\title{
A Reciprocity Theorem for Monomer-Dimer Coverings
}

\author{
Nick Anzalone ${ }^{1}$ and John Baldwin ${ }^{2}$ and Ilya Bronshtein ${ }^{3}$ and T. Kyle \\ Petersen $^{4}$ \\ ${ }^{1}$ University of Massachussetts-Boston, Boston, MA, USA, njanzalo@cs.umb.edu \\ ${ }^{2}$ Harvard University, Cambridge, MA, USA, jbaldwin@fas.harvard.edu \\ ${ }^{3}$ Brandeis University, Waltham, MA, USA, ilyabron@brandeis.edu \\ ${ }^{4}$ Department of Mathematics, Brandeis University, Waltham, MA, USA, tkpeters@brandeis.edu
}

\begin{abstract}
The problem of counting monomer-dimer coverings of a lattice is a longstanding problem in statistical mechanics. It has only been exactly solved for the special case of dimer coverings in two dimensions ((Kas61), (TF61)). In earlier work, Stanley (Sta85) proved a reciprocity principle governing the number $N(m, n)$ of dimer coverings of an $m$ by $n$ rectangular grid (also known as perfect matchings), where $m$ is fixed and $n$ is allowed to vary. As reinterpreted by Propp (Pro01), Stanley's result concerns the unique way of extending $N(m, n)$ to $n<0$ so that the resulting biinfinite sequence, $N(m, n)$ for $n \in \mathbb{Z}$, satisfies a linear recurrence relation with constant coefficients. In particular, Stanley shows that $N(m, n)$ is always an integer satisfying the relation $N(m,-2-n)=\varepsilon_{m, n} N(m, n)$ where $\varepsilon_{m, n}=1$ unless $m \equiv 2(\bmod 4)$ and $n$ is odd, in which case $\varepsilon_{m, n}=-1$. Furthermore, Propp's method was applicable to higherdimensional cases. This paper discusses similar investigations of the numbers $M(m, n)$, of monomer-dimer coverings, or equivalently (not necessarily perfect) matchings of an $m$ by $n$ rectangular grid. We show that for each fixed $m$ there is a unique way of extending $M(m, n)$ to $n<0$ so that the resulting bi-infinite sequence, $M(m, n)$ for $n \in \mathbb{Z}$, satisfies a linear recurrence relation with constant coefficients. We show that $M(m, n)$, a priori a rational number, is always an integer, using a generalization of the combinatorial model offered by Propp. Lastly, we give a new statement of reciprocity in terms of multivariate generating functions from which Stanley's result follows.
\end{abstract}

Keywords: reciprocity, monomer-dimer coverings, linear recurrences

\section{Introduction}

\subsection{Background}

The problem of counting the number of monomer-dimer coverings of a lattice has been examined for many years in the fi eld of statistical mechanics (see (KRS96)), and has applications in biology, chemistry and physics. The closely related dimer problem, where the number of monomers is zero, was exactly solved for two dimensional lattices by Kasteleyn (Kas61) and Temperley and Fisher (TF61). While we cannot offer an exact solution to the monomer-dimer problem, we can express a symmetry relationship between the numbers of coverings of certain families of lattices; the base case being a rectangular grid of 1365-8050 (c) 2003 Discrete Mathematics and Theoretical Computer Science (DMTCS), Nancy, France 
fi xed height and varying width. Our results are not limited to two dimensions, however our families are classifi ed by their growth in only one dimension.

In particular, we consider families of lattices indexed by the natural numbers $(n=1,2,3, \ldots)$, and count the numbers, $a_{n}$, of monomer-dimer coverings on the lattices. The numbers $a_{n}$ satisfy a linear recurrence which we can use to compute the numbers $a_{0}, a_{-1}, a_{-2}, \ldots$ Surprisingly, we fi nd that the new numbers are related, term by term, to the original sequence of numbers; for instance $a_{n} \sim a_{-n-2}$. Richard Stanley's book (Sta97), in the context of rational generating functions, devotes an entire section to exploring the relationships (called reciprocity relationships) between positively- and nonpositively-indexed terms of a sequence. The nonpositively-indexed terms may even have a nice combinatorial meaning on their own, as seen in the case of Ehrhart reciprocity, for example. ${ }^{\dagger}$ In this paper we describe a relationship between terms of certain integer sequences $\left\{a_{n}\right\}, n \in \mathbb{Z}$, as well as give a combinatorial interpretation to the nonnatural terms.

\subsection{The Problem}

Rather than use the language of statistical mechanics (monomers and dimers on lattices), we will refer to matchings of graphs where we defi ne a matching of a graph $G=(V, E)$ to be a collection of edges of $G$, no two of which share a vertex, and all the vertices that are not incident with those edges. ${ }^{\ddagger}$ Edges correspond to dimers, isolated vertices correspond to monomers. A matching is called perfect if it is composed entirely of edges, as in the dimer problem. If we consider the number of matchings of a rectangular gridgraph of fi xed height $m$, and varying width $n$, we obtain an integer sequence, $\left\{a_{n}\right\}$. For example, when $m=1$, we obtain the Fibonacci sequence:

$$
1,2,3,5,8, \ldots
$$

This sequence satisfi es a linear recurrence, namely $a_{n}=a_{n-1}+a_{n-2}$. So we can always obtain the value of a term based on the two terms to the left of it. But similarly, we can obtain the value of a term by the two terms to the right of it, e.g. $3=8-5$, or $a_{n}=a_{n+2}-a_{n+1}$. In this way we can extend the Fibonacci sequence to the left and get values for $a_{n}$ when $n \leq 0$ :

$$
\ldots-8,5,-3,2,-1,1,0,1,1,2,3,5,8, \ldots
$$

So we see that we now have a doubly infi nite integer sequence, and that it is symmetric up to sign, i.e. $a_{n}= \pm a_{-n-2}$. Some natural questions arise: Does this symmetry mean something? Do the values of $a_{n}$ for $n<0$, being integers, count something? If so, can we extend the result to larger values of $m$ ?

The answer to all the questions is yes. In (Pro01), Propp considered integer sequences $N(m, n)$, generated by perfect matchings of rectangular grid graphs of fi xed height, and how they extend to values for $n<0 .{ }^{\S}$ He came up with a unifi ed combinatorial object and a way of counting signed matchings of these objects that allowed him to show that any half-infi nite sequence given by the number of perfect matchings of a fi xed-height grid graph extends to a bi-infi nite sequence that does three things:

- It satisfi es a linear recurrence relation of fi nite degree with constant coeffi cients.

\footnotetext{
$\dagger$ Ehrhart reciprocity describes a relationship between the number of lattice points found in a rational polytope, and the number of lattice points found in its interior. See (Sta97)

\# Another equivalent terminology would be to speak of tilings of planar regions where the tile set consists of a domino ( 2 by 1 rectangle) and a square ( 1 by 1 rectangle).

$\S$ His fi rst nontrivial case, $N(2, n)$ is also the Fibonacci sequence.
} 
- The numbers obtained when going backwards are unique and are always integers.

- The bi-infi nite sequences have a special kind of symmetry, or reciprocity, stated roughly as $|N(m, n)|=$ $|N(m,-n-2)|$ for any $m>0$.

Matchings in general (of the graphs we will classify) satisfy the same sort of linear recurrence (as we will explain in section 3). But even given that a covering problem satisfi es a linear recurrence, integrality is not ensured when running the recurrence backwards. ${ }^{\text {II }}$ For a simple example, consider covering a 2-by- $n$ grid with monomers and dimers, but only allow the dimers to be vertical. The number of such coverings is governed by

$$
a_{n}=2 a_{n-1} .
$$

Upon observing that $a_{1}=2$, we can generate the half-infi nite sequence

$$
2,4,8,16, \ldots
$$

To work the sequence backwards, we invert the recurrence:

$$
a_{n}=\frac{1}{2} a_{n+1}
$$

to generate the bi-infi nite sequence

$$
\ldots \frac{1}{8}, \frac{1}{4}, \frac{1}{2}, 1,2,4,8, \ldots
$$

For $n=-1$ we cease to observe integrality. However in the case of monomer-dimer tilings, we can guarantee integrality of the sequences by showing they can be generated by counting matchings of certain graphs for all values of $n$.

The objects we will present not only ensure integrality, but also give a direct proof of the paper's main idea: a reciprocity statement for general matchings. This claim would appear to be the least obvious judging by the numerical evidence. Indeed, consider the integer sequence $M(2, n)$, the number of matchings of a 2-by- $n$ rectangular grid graph. The sequence is governed by the recurrence

$$
M(2, n)=3 M(2, n-1)+M(2, n-2)-M(2, n-3)
$$

with initial 'natural' values $M(2,1)=2, M(2,2)=7$, and $M(2,3)=22$, allowing us to generate the bi-infi nite sequence

$$
\ldots 14,11,2,3,0,1,0,1,2,7,22,71,228, \ldots
$$

Although we get integers when going backwards, we seem to lack the symmetry that Stanley and Propp observed for perfect matchings. However section 4 will reveal that there is a symmetry hidden in these numbers.

Why there should be symmetry in integer sequences as above is explained in the answer to the question: what do the numbers, $M(m, n)$ for $n<0$, count? Propp had an answer for the case of perfect matchings, but it doesn't directly translate to general matchings. Section 2 outlines the dilemma and gives a solution

II A priori the numbers obtained from running a linear recurrence in reverse need only be rational. If the recurrence is of the form $a_{n}=c_{1} a_{n-1}+c_{2} a_{n-2}+\cdots+c_{k} a_{n-k}$, then to push the sequence backwards we solve for the smallest term to get $a_{n-k}=$ $\frac{a_{n}-c_{1} a_{n-1}-\cdots-c_{k-1} a_{n-k+1}}{c_{k}}$ 
by way of objects called "empty vertices". In Propp's article (Pro01), weights were not given to isolated vertices, simply because there were none. But when examining matchings as we've defi ned them (a monomer-dimer model), isolated vertices do have weights, giving rise to the need for empty vertices to explain the observed phenomenon and tell the "proper" combinatorial story. By incorporating empty vertices we will obtain a combinatorial model whose number of signed matchings is $M(m, n)$ for all $n$. Looking at the signed matchings we can see the symmetry hidden in the terms above:

$$
\ldots 121-107,41-30,12-10,5-2,1-1,1,0,1,1+1,5+2,12+10,41+30,121+107, \ldots
$$

The properties exhibited by the numbers $M(m, n)$ and the objects they count can also be seen in terms of the generating function $F_{m}(t, x, y, z)=\sum_{n=1}^{\infty} f_{n}(x, y, z) t^{n}$. Here $x$ is the weight given to horizontal edges, $y$ is the weight given to vertical edges, and $z$ is the weight given to vertices. The polynomial $f_{n}(x, y, z)$ encodes all of the matchings of an $m$ by $n$ grid graph. We will show

$$
\begin{aligned}
& \text { 1. } F_{m}(t, 1,1,1)=\sum_{n=1}^{\infty} M(m, n) t^{n} \\
& \text { 2. } F_{m}(t, 1,1,0)=\sum_{n=1}^{\infty} N(m, n) t^{n} \\
& \text { 3. } F_{m}(t, x, y, z) \sim-F_{m}(1 / t, x,-y,-z)
\end{aligned}
$$

The second item in the list shows how perfect matchings may be extracted from general matchings. The last item in the list is a heuristic expression of reciprocity that will be made precise in section 4.2.

The theory of empty vertices is applicable to more general situations than simply matchings of rectangular grid graphs. We will prove results for matchings of graphs of the form $G \times P_{n}$, where $G$ is an arbitrary fi nite graph and $P_{n}$ is the path graph of length $n$. We will refer to such graphs as "generalized rectangles". Notice that an $m$ by $n$ rectangular grid graph is $G \times P_{n}$ where $G=P_{m}$. If $M(n)$ is the number of matchings of $G \times P_{n}$, we can show that there are objects whose number of matchings is $M(-n)$, i.e. a defi nition for $G \times P_{-n}$. By extension, this implies that the same symmetry will exist for the entire family of generalized rectangles. Throughout most of the paper, our proofs will be for generalized rectangles, but examples will usually involve grid graphs.

\section{Signed Graphs and Signed Matchings}

Let us try to deduce the nature of $m$ by $n$ rectangular grid graphs for all $n$, not concerning ourselves with matchings at all. In Figure 1, denote the 2 by 3 grid graph by $G(2,3)$. The graph $G(2,4)$ is obtained from $G(2,3)$ by adding two horizontal edges, a vertical edge, and two vertices as shown. Likewise, we can obtain $G(2,2)$ by removing the same set of edges and vertices from the right of $G(2,3)$. Then we get $G(2,1)$ and $G(2,0)$ inductively. But what are we to make of $G(2,0)$ ? It has no vertices, and two "anti-" horizontal edges. How many matchings should this have? What about $G(2,-1)$ ? $G(2,-2)$ ?

The defi nitions that Propp created for $G(m, n)$ with $n<0$ look quite similar to the pictures in Figure 1 , except that his horizontal lines are solid and there are vertices on the endpoints of the horizontal edges where there are none in Figure 1 (see Figure 2).

Notice that in Figure 1 the graphs for $G(2,0)$ and $G(2,-1)$ have some horizontal edges that are incident with only one vertex. The meaning of these missing vertices, these places that are "empty" of vertices, is described below. Let $G=(V, E)$ be a graph in the usual sense, i.e. a set of vertices and edges between them, except that there is more than one kind of edge and more than one kind of vertex (see Figure 3 ). 


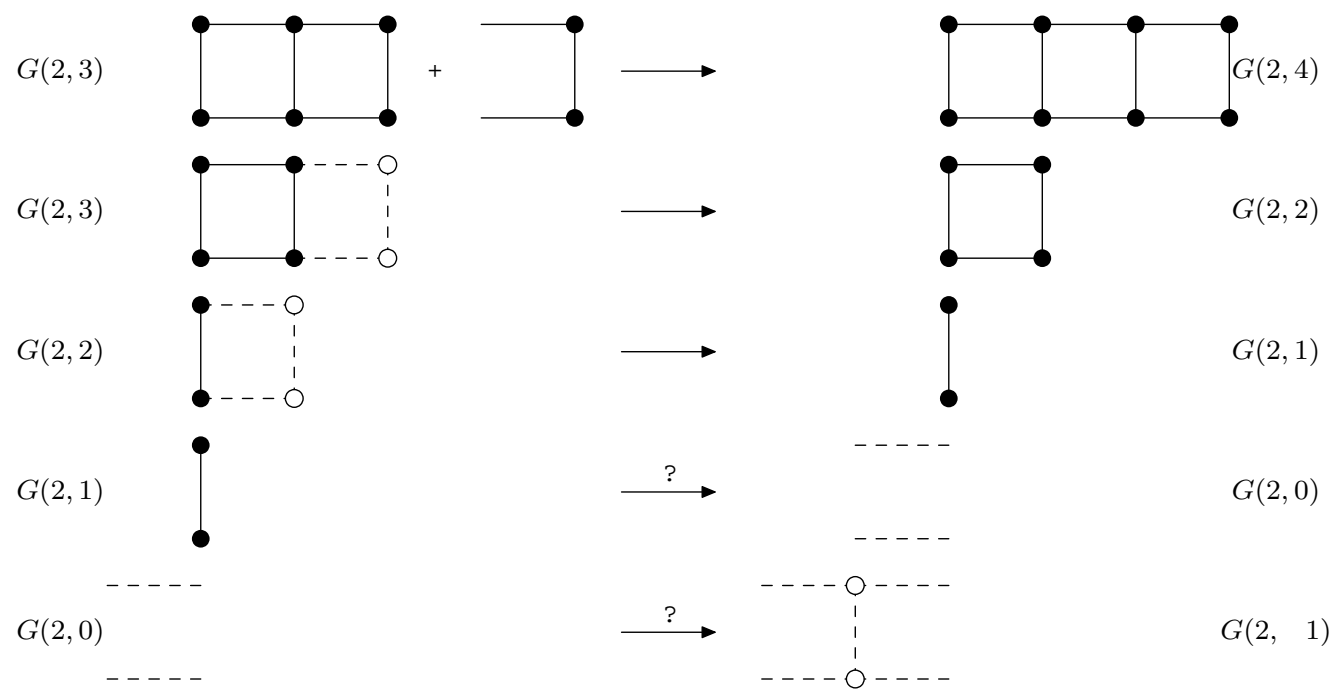

Fig. 1: Moving from $G(2,3)$ to $G(2,4)$ or $G(2,2)$

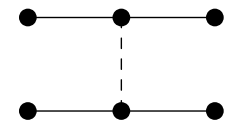

Fig. 2: Propp's model for $G(2,-1)$.

There are plain, anti-, and empty vertices, plain and anti-vertical edges (vedges), and plain horizontal edges (hedges). For now, plain components are given weight 1 , anti-vertices and anti-vedges are given weight -1 , and empty vertices have weight zero."

$$
\text { Plain Anti Empty }
$$

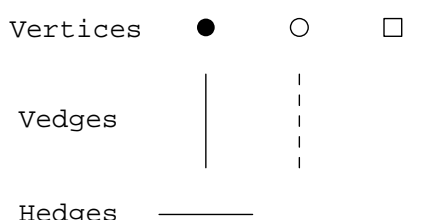

Fig. 3: Basic components of a signed graph.

Defi ne a signed matching of a graph $G$ to be a collection of non-adjacent edges of $G$ and all the vertices not incident with those edges. The weight of the matching is the product of the weights of the components in the matching. Note that this means all the matchings with empty vertices vanish. In practice, it helps

" Later we will attach formal variables to edges and vertices, but empty vertices will still have weight zero. Thinking of monomerdimer coverings, empty vertices represent positions in the lattice that may not be occupied by a monomer. 
to think of empty vertices as being needy. For any nonzero matching, empty vertices need to have one of their incident edges included.

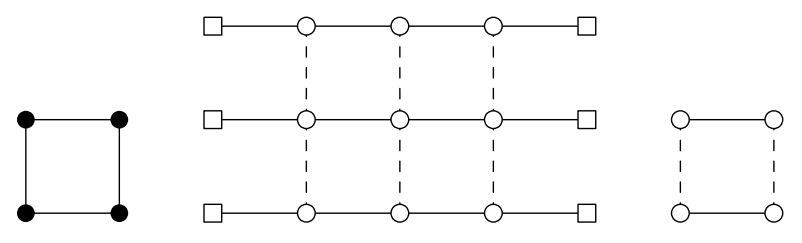

Fig. 4: Pictures of graphs $G(2,2), G(3,-3)$, and $G^{*}(2,2)$.

Defi ne a regular signed graph, $G(m, n)=P_{m} \times P_{n}$ in the following way. If $n>0, G(m, n)$ is just a rectangular grid graph, i.e., a graph with $m n$ plain vertices arranged into $m$ rows of $n$ vertices each, with plain edges adjoining horizontal and vertical neighbors. Defi ne the conjugate graph of $G$, denoted $G^{*}$, to be the graph obtained by replacing all of the vertices and vedges of $G$ with their anti-counterparts (assuming that an anti-anti-vertex is a plain vertex, etc.). Under this defi nition, $(G(m, n))^{*}=G^{*}(m, n)$ is a graph with $m n$ anti-vertices arranged into $m$ rows of $n$ vertices with hedges adjoining horizontal neighbors and anti-vedges adjoining vertical neighbors. Then for $n \leq 0, G(m, n)$ is defi ned to be a copy of $G^{*}(m, n)$ with a column of $m$ empty vertices on the left and $m$ empty vertices on the right. Each empty vertex is connected (with a hedge) only to the anti-vertex horizontally adjacent to it (see Figure 4).

For fi xed $m>0$ and any integer $n$, we defi ne the numbers $M(m, n)=M(G(m, n))$ to be the sum of the weights of the signed matchings of $G(m, n)$. In general, $M(G)$ is the sum of the weights of all of the signed matchings of $G$. Some examples are given in Figure 5.

Having made all our defi nitions in terms of rectangular grid graphs, we would now like to extend them to apply to general rectangular regions. For any fi nite graph $G$, defi ne the generalized rectangle graph $G \times P_{n}$ with $n>0$ as follows: Picture $n$ copies of $G$ lined up side by side. Each vertex in one copy of $G$ is connected with an edge to its image in the copies of $G$ to the left and right of it. Defi ne all the edges within a copy of $G$ to be vedges, and the edges between copies of $G$ to be hedges. With this idea, we can defi ne $G \times P_{-n}$ to be $G^{*} \times P_{n}$ with $m$ (= the number of vertices in $G$ ) empty vertices to the left and to the right of it. Each empty vertex is connected to exactly one of the vertices of $G^{*}$ with a hedge. We shall see that these defi nitions are necessary to prove statements of reciprocity for more than just grid graphs.

\section{Adjunction}

Although it seems there are separate defi nitions for $G(m, n)$ (resp. $G \times P_{n}$ ) when $n>0$ and when $n \leq 0$, we will show they are actually the same object. In Propp's words, they fi t together "seamlessly." If they fi t together this way, then it follows that for any $G$, the sequence $M\left(G \times P_{n}\right)$ satisfi es a linear recurrence of fi nite degree with constant coeffi cients. We will omit the proof as it is given in (Pro01). The method of proof utilizes transfer matrix methods and the Cayley-Hamilton theorem, and it requires only that the family of graphs satisfy the property detailed below.

We begin by making a natural observation about the half-infi nite sequence $M(G(m, n)), n>0$, and then we will prove that it actually holds for any $n$. In doing so, we will be able to establish that we do indeed have an appropriate defi nition for $G(m, n)$ (resp. $G \times P_{n}$ ) with $n \leq 0$, and all our desires for the doubly infi nite sequence—integrality, uniqueness, and reciprocity—will be fulfi lled. 


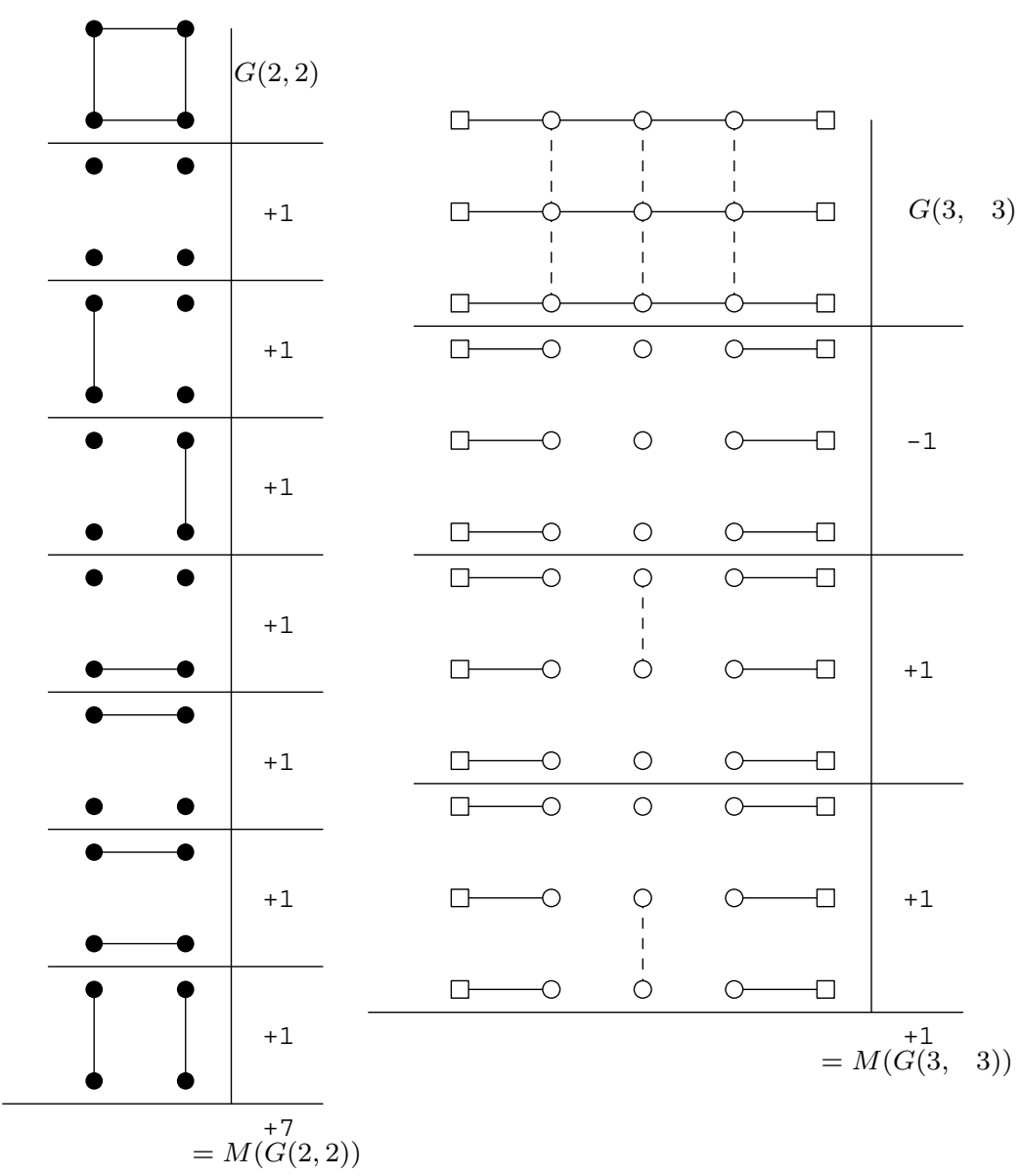

Fig. 5: Pictures of nonzero signed matchings of $G(2,2)$ and $G(3,-3)$.

We fi rst notice that for positive $n_{1}, n_{2}$, we can draw the graph $G\left(m, n_{1}+n_{2}\right)$ by placing $G\left(m, n_{1}\right)$ and $G\left(m, n_{2}\right)$ side by side and using hedges to connect the rightmost $m$ vertices of one to the leftmost $m$ vertices of the other, as shown in Figure 6. Call this operation adjunction. Generally, defi ne the adjunction of two graphs $H=G \times P_{n_{1}}, H^{\prime}=G \times P_{n_{2}}$ to be a new graph formed by connecting $H$ to $H^{\prime}$ with hedges, matching up corresponding vertices. In particular:

- If $n_{1}, n_{2}$ are positive, connect every vertex in the rightmost copy of $G$ in $H$ to its image in the leftmost copy of $G$ in $H^{\prime}$ using a hedge.

- If $n_{1}$ is positive, $n_{2}$ negative, join every vertex $v$ in the rightmost copy of $G$ in $H$ to an empty vertex on the left side of $H^{\prime}$ so that $v$ is connected to $v^{*}$ (the anti-vertex corresponding to $v$ ) in the leftmost copy of $G^{*}$ in $H^{\prime}$ by the path hedge-empty vertex-hedge.

- If $n_{1}, n_{2}$ are both negative, join every vertex in the rightmost copy of $G^{*}$ in $H$ to its image in the 

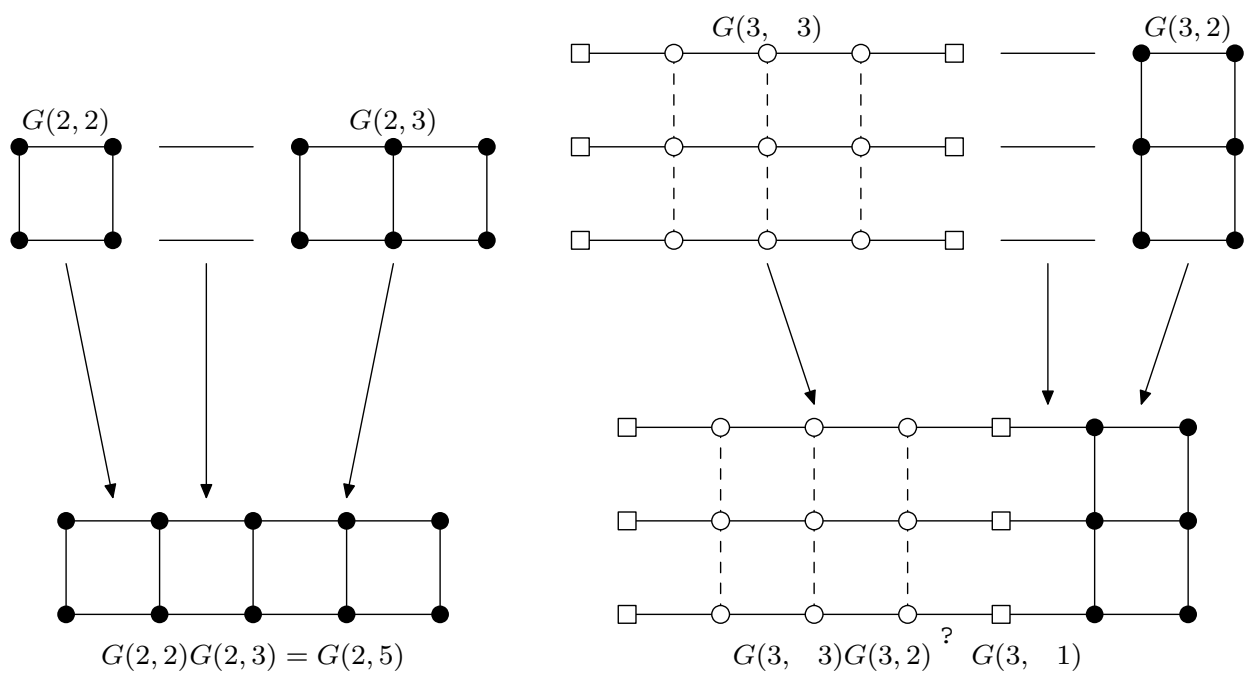

Fig. 6: Pictures of two pairs of adjoined graphs.

leftmost copy of $G^{*}$ in $H^{\prime}$ by connecting their adjacent empty vertices with a hedge.

We write the adjunction of $G \times P_{n_{1}}$ and $G \times P_{n_{2}}$ as $\left(G \times P_{n_{1}}\right)\left(G \times P_{n_{2}}\right)$.

Returning to case of rectangular grid graphs where $n_{1}, n_{2}$ are positive, $G\left(m, n_{1}\right) G\left(m, n_{2}\right)=G\left(m, n_{1}+\right.$ $\left.n_{2}\right)$, and naturally, $M\left(G\left(m, n_{1}\right) G\left(m, n_{2}\right)\right)=M\left(G\left(m, n_{1}+n_{2}\right)\right)$. This is true more generally, as stated in the following:

\section{Theorem 1 (Adjunction)}

$$
M\left(\left(G \times P_{n_{1}}\right)\left(G \times P_{n_{2}}\right) \cdots\left(G \times P_{n_{k}}\right)\right)=M\left(G \times P_{n_{1}+n_{2}+\cdots+n_{k}}\right)
$$

for all integers $n_{1}, \ldots, n_{k}$. In particular,

$$
M\left(G\left(m, n_{1}\right) G\left(m, n_{2}\right) \cdots G\left(m, n_{k}\right)\right)=M\left(G\left(m, n_{1}+n_{2}+\cdots+n_{k}\right)\right)
$$

for all integers $n_{1}, \ldots, n_{k}$.

The proof of Theorem 1 will require the following two lemmas.

Lemma 1 Let $G$ be a signed graph where two vertices, a and b, are connected by the path hedge-empty vertex-hedge-empty vertex-hedge. Then $G$ has same number of matchings as the graph $G^{\prime}$, where $G^{\prime}$ is identical to $G$ except that $a$ and $b$ are connected with one hedge.

Proof: The claim made by Lemma 1 is most easily seen in Figure 7 where we see the immediate bijection. When the hedge between the empty vertices is present in $G$, then in any matching $a$ and $b$ are not connected inwards. This corresponds to the case when the hedge between $a$ and $b$ in $G^{\prime}$ is not present.

On the other hand, any matching of $G$ where the hedge between the empty vertices is not present forces $a$ and $b$ to be connected inwards. This is equivalent to the hedge between $a$ and $b$ being present in a matching of $G^{\prime}$. 


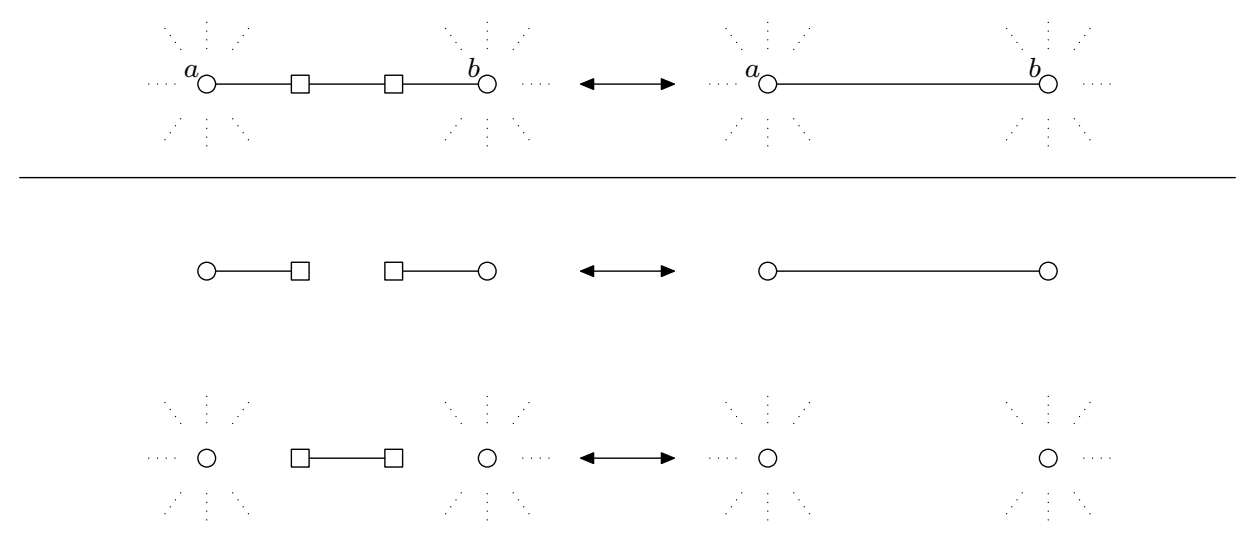

Fig. 7: Proof of bijection between $G$ and $G^{\prime}$ in Lemma 1 .

Lemma 2 Let $G$ be a finite graph with all plain components. Then let $H$ be the graph $\left(G \times P_{n_{1}}\right)\left(G \times P_{n_{2}}\right)$ for $n_{1}>0>n_{2}$. Then $H$ has the same number of signed matchings as the graph $H^{\prime}=\left(G \times P_{n_{1}-1}\right)(G \times$ $\left.P_{n_{2}+1}\right)$.

Proof: We begin by examining what happens at each vertex, see Figure 8. Consider the plain vertex $a$ of $G$ connected to the anti-vertex $a^{*}$ of $G^{*}$ by the path hedge-empty vertex-hedge. There are essentially two types of matchings of $H$. We'll say type 1 matchings include the hedge connecting $a$ to the empty vertex and type 2 matchings include the hedge connecting $a^{*}$ to the empty vertex. Most of the matchings of type 1 will cancel with most of the matchings of type 2 .

Given a matching of type 1 , there are only three cases for what can happen the anti-vertex $a^{*}$ of $G^{*}$ : it can be isolated, it can have an anti-vedge incident with it, or it can have a hedge incident with it. Likewise for any matching of type 2, the vertex $a$ can be isolated, incident with a vedge or incident with a hedge. If there are $k$ signed matchings of type 1 where $a^{*}$ is isolated, then there are $-k$ signed matchings of type 2 where $a$ is isolated. Similar cancellation occurs between matchings of type 1 where $a^{*}$ has an anti-vedge and matchings of type 2 where $a$ has a vedge (corresponding to the anti-vedge incident with $a^{*}$ ).

The only remaining cases are those of type 1 and type 2 where $a$ and $a^{*}$ both have hedges. We claim that these matchings are in bijection with the graph where $a, a^{*}$, all their incident vedges, and the hedges and empty vertex between $a, a^{*}$ are all replaced with one empty vertex. The correspondence is shown in Figure 8. Since $a$ was any vertex of $G$, this can be done for every vertex of $G$ and the lemma holds.

With the lemmas proved, Theorem 1 is not diffi cult to show.

Proof of Theorem 1: It is easily verifi ed that $M\left(\left(G \times P_{0}\right)\left(G \times P_{n}\right)\right)=M\left(G \times P_{n}\right)$, so we may assume all the $n_{i}$ are non-zero. We have that $M\left(\left(G \times P_{n_{1}}\right)\left(G \times P_{n_{2}}\right)\right)=M\left(G \times P_{n_{1}+n_{2}}\right)$ whenever $n_{1}$ and $n_{2}$ are negative by applying Lemma 1 to each place where adjoining takes place. When $n_{1} n_{2}<0$, we can apply Lemma 2 repeatedly. Upon each application of Lemma 2 we change neither the number of matchings nor the difference between the number of vertices and the number of anti-vertices. 

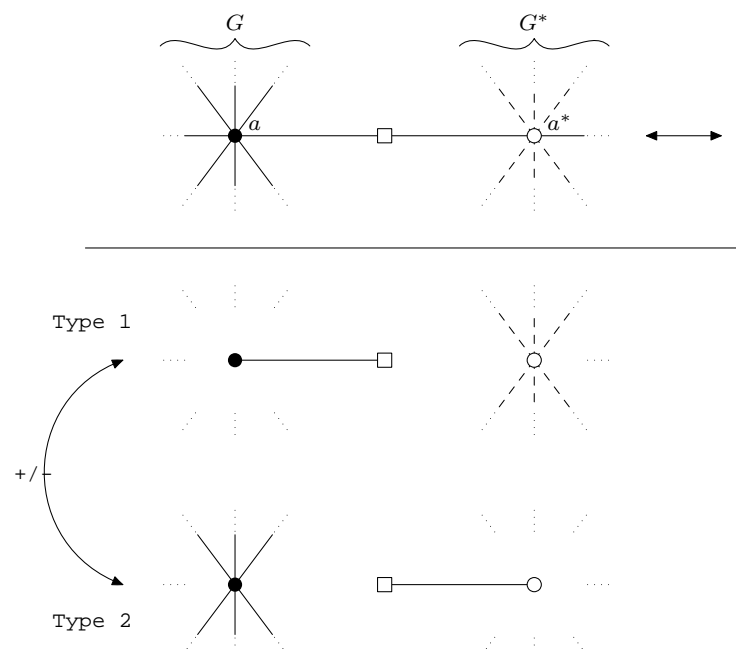

Type 1
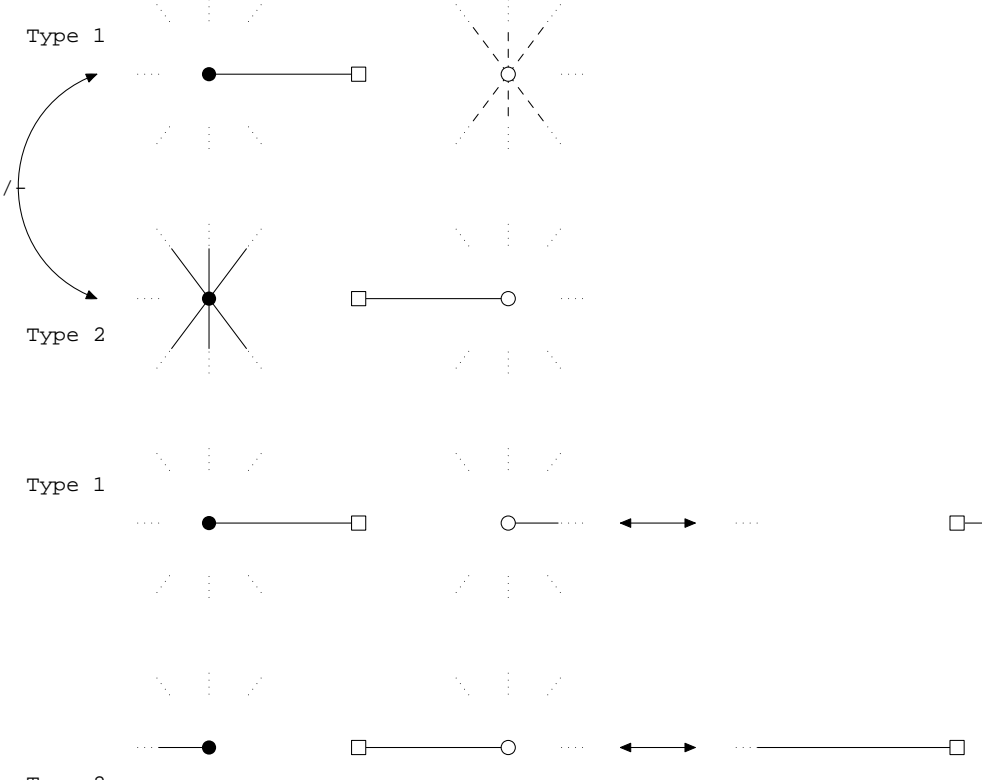

Fig. 8: Proof of Lemma 2.

\section{Reciprocity}

\subsection{Combinatorial Reciprocity}

The combinatorial statement of reciprocity is rather obvious given the defi nition of $G \times P_{n}$ for $n \leq 0$ and the neediness of empty vertices:

\section{Theorem 2 (Reciprocity (I))}

$$
M\left(G \times P_{-n-2}\right)=M\left(G^{*} \times P_{n}\right) .
$$

In particular,

$$
M(G(m,-n-2))=M\left(G^{*}(m, n)\right) .
$$

Proof: On the left side of Figure 10 we have $G(m,-n-2)$. But since all the outside edges are forced, the number of matchings of $G(m,-n-2)$ is clearly equal to the number of matchings of $G^{*}(m, n)$. The situation for generalized rectangles is identical. 


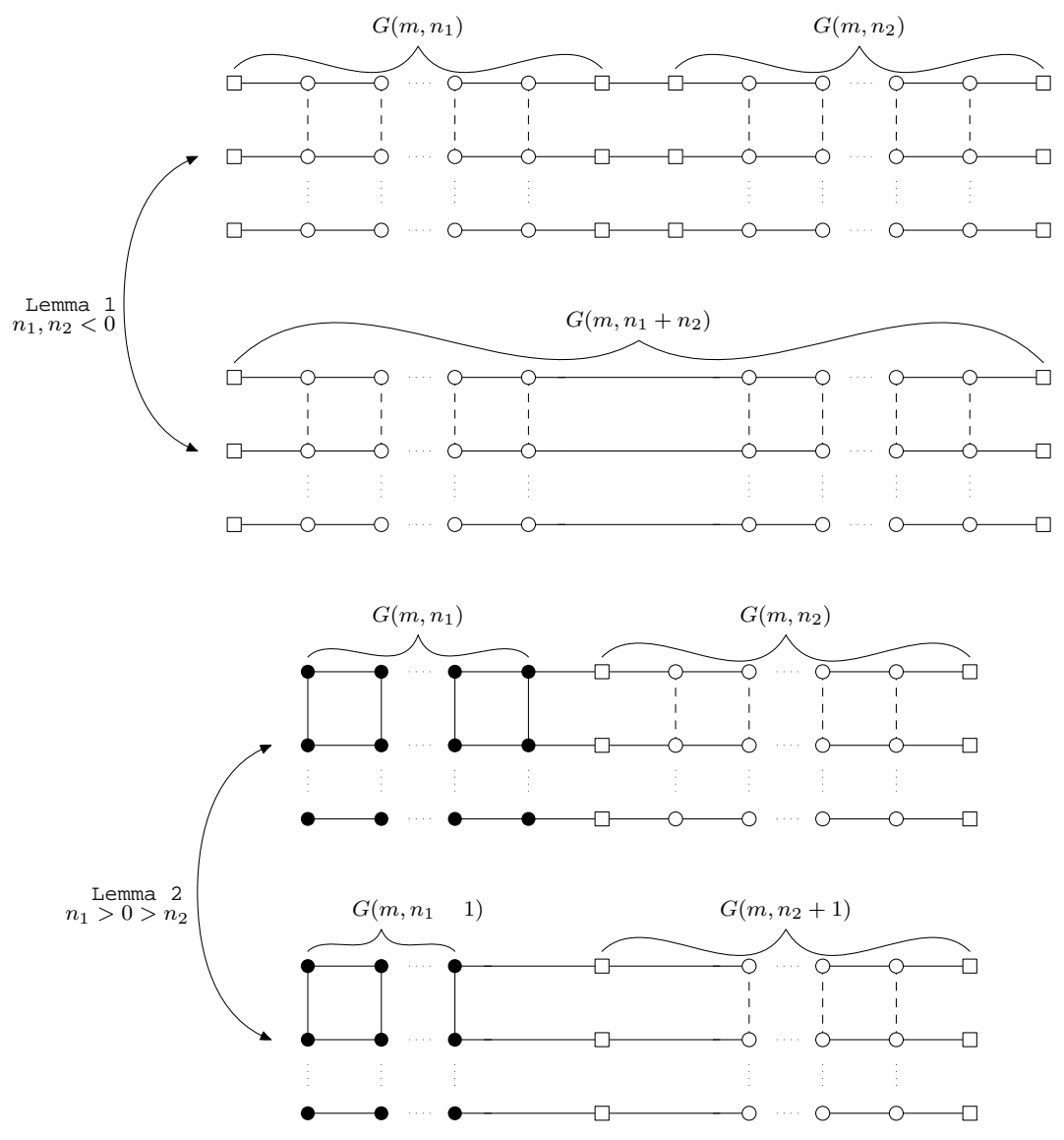

Fig. 9: Picture of Theorem 1 using lemmas.

This statement of reciprocity is the natural extension of the property of dimer coverings observed by Propp (Pro01). Indeed, if we were to consider only the perfect matchings of grid graphs, then the statement would be the same:

$$
N(G(m,-n-2))=N\left(G^{*}(m, n)\right)
$$

It is known that any dimer covering can be obtained from any other dimer covering by local moves that leave the parity of vedges unchanged. That is, the sign of every perfect matching of $G^{*}(m, n)$ is the same, so $|N(m, n)|=\left|N^{*}(m, n)\right|$. With less-than-perfect matchings, this is clearly not the case.

Perhaps the strength of this result is more obvious when we give all the edges and vertices formal weights and derive a statement of reciprocity in terms of a generating function. Though we could do so for any fi nite graph of the form $G \times P_{n}$, we will only derive an explicit formula for $G(m, n)$. 

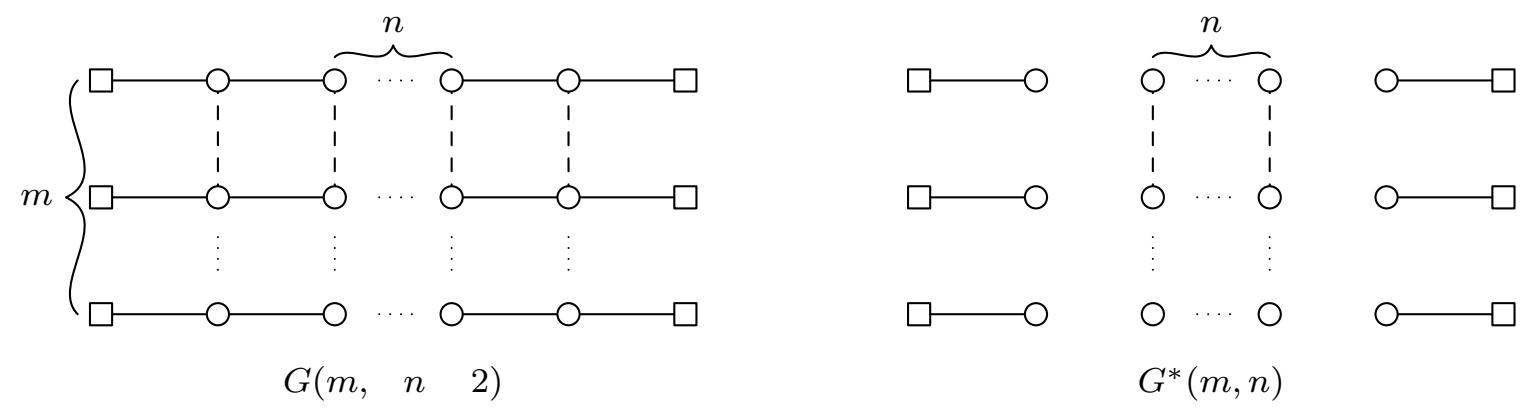

Fig. 10: Picture of Reciprocity for grid graphs.

\subsection{The Generating Function}

For convenience, let the weights of the vertices of $G(m, n)$ for $n>0$ be indexed by $\mathbb{N} \times \mathbb{N}$, read from left to right and top to bottom. Notice that the top left vertex is $z_{1,1}$ and the bottom right vertex is $z_{m, n}$. Then $x_{i, j}$ is the weight given to the hedge between $z_{i, j}$ and $z_{i, j+1}$, and $y_{i, j}$ is the weight given to the vedge between $z_{i, j}$ and $z_{i+1, j}$. To get the weights for vertices of $G(m,-n)$, we begin by giving the top right anti-vertex weight $z_{1,0}$ and we proceed analogously. The weights of $G(2,3)$ and $G(2,-5)$ are shown in Figure 11.

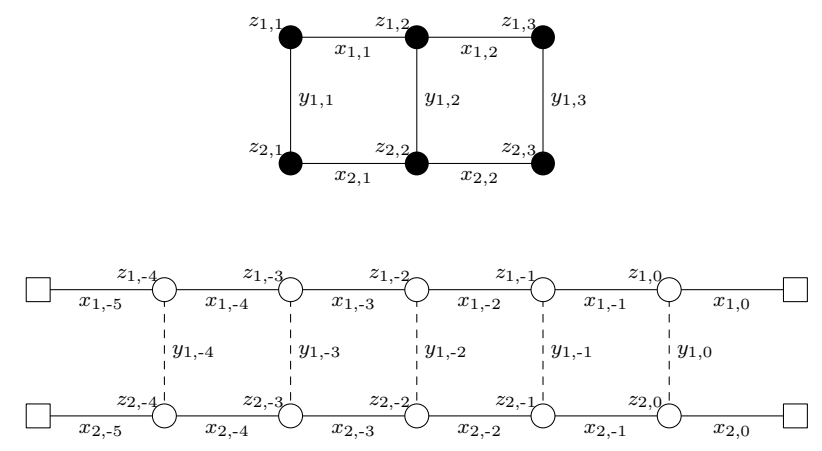

Fig. 11: Picture of graphs weighted with formal variables.

The weight of a matching with formal variables is not exactly like the weight as defi ned before, as is seen in the following:

Definition 1 If $g$ is a matching of $G(m, n)$, define the weight of $g, w(g)$, as follows (where $\operatorname{sgn}(t)=1$ if $t$ is positive, -1 otherwise):

$$
w(g)=\frac{\left(\prod_{\text {hedges in } g} x_{i, j}\right)\left(\prod_{\text {vedges in } g} \operatorname{sgn}(j) y_{i, j}\right)\left(\prod_{\text {vertices in } g} \operatorname{sgn}(j) z_{i, j}\right)}{\prod_{\text {all hedges in } G(m, n)} x_{i, j}^{(1-\operatorname{sgn}(j)) / 2}} .
$$


Notice that for $n<0$, there is a monomial denominator in the weights of matchings. By our defi nition, the matchings shown in Figure 12 have weights

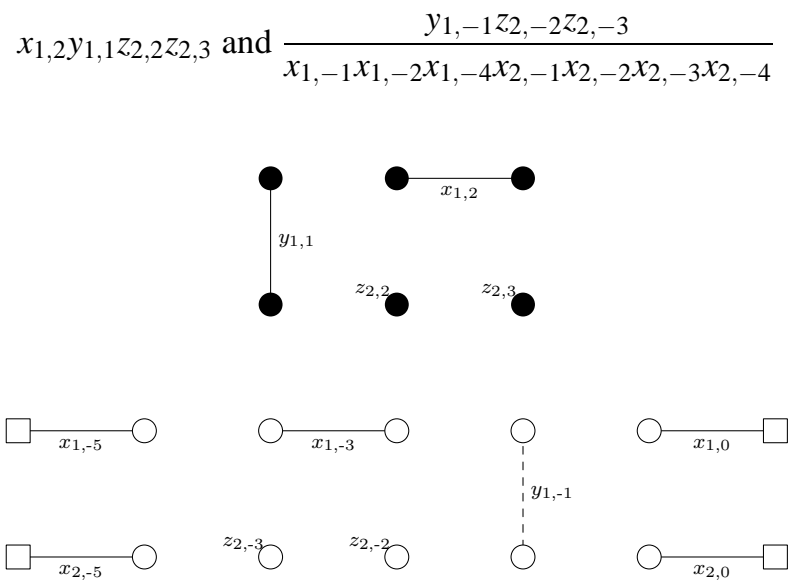

Fig. 12: Picture of matchings weighted with formal variables.

With this new defi nition of weights, we defi ne the matching polynomial. In the literature, there are conventions for describing the matching polynomial of a graph. Our defi nition does not adhere strictly to these conventions, though it is similar to the partition function for the monomer-dimer model, with distinct formal variables for all monomers and dimers (see (HL72),(COSW02)).

Definition 2 The matching polynomial of $G(m, n), f_{n}\left(x_{i, j}, y_{i, j}, z_{i, j}\right)$, is given by

$$
f_{n}\left(x_{i, j}, y_{i, j}, z_{i, j}\right)=\sum_{\text {all matchings of } G(m, n)} w(g)
$$

where $i=1 \ldots m, j=1 \ldots n$ if $n>0, j=0,-1 \ldots n$ if $n<0$,

Strictly speaking, the matching polynomial is a Laurent polynomial; a polynomial in the variables $x_{i, j}$, $x_{i, j}^{-1}, y_{i, j}, z_{i, j}$. Notice if we set all of the weights equal to 1 we get $f_{n}(1,1,1)=M(G(m, n))$, the number of signed matchings. Similarly if we set all the $x_{i, j}, y_{i, j}$ equal to 1 but set all the $z_{i, j}$ equal to 0 , then $f_{n}(1,1,0)=N(G(m, n))$, the number of signed perfect matchings.

By construction the polynomials $f_{n}$ will satisfy a linear recurrence very similar to that which governs $M(G(m, n))$. For example, if $m=1$, then we get $f_{n}=z_{1, n} f_{n-1}+x_{1, n-1} f_{n-2}$ for all $n$. Likewise, there is a link between the polynomials $f_{n}$ and $f_{-n-2}$ for $n \geq 0$, seen most easily if we let $x_{i, j}=x, y_{i, j}=y, z_{i, j}=z$ for all $i, j$ :

$$
f_{n}(x,-y,-z)=x^{m(n+1)} f_{-n-2}(x, y, z) .
$$

As mentioned in Section 3, for any fi xed $m$, the numbers $M(m, n), n \in \mathbb{Z}$ satisfy a linear recurrence of fi nite degree with constant coeffi cients. Therefore there is a rational generating function for the number of weighted matchings,

$$
F_{m}(t, x, y, z)=\sum_{n \geq 0} f_{n}(x, y, z) t^{n}
$$


Given (1) it is an easy exercise (see for example (Sta97), ch. 4) to state a reciprocity theorem for the generating function:

Theorem 3 (Reciprocity(II))

$$
x^{m} t^{2} F_{m}(t, x, y, z)=-F_{m}\left(\frac{1}{t x^{m}}, x,-y,-z\right) .
$$

This completes the main goal of the paper.

\section{More on Linear Recurrences and Reciprocity}

Though we have only proved theorems here for graphs of the type $G \times P_{n}$, (cylinders of fi xed circumference and varying height for example), we have also been able to use a modifi ed form of adjunction to build a model for the honeycomb graph of fi xed height and width $n \in \mathbb{Z}$. Other graphs that we examined but were unable to apply our methods include: cylinders with fi xed height and varying circumference, Möbius strips, tori, and projective planes. We do however feel that there may be a way of dealing with such graphs.

In an unpublished paper (Spe02), Speyer developed a matrix method for encoding perfect matchings of a graph. With this method he was able to state theorems about recurrences and reciprocity for a broader range of graphs than those we have handled here. In particular, he was able to make a statement about the Möbius strip and projective plane. An adaptation of his method to general matchings seems promising, though experimentation has shown it to be less than straightforward.

\section{Acknowledgements}

We'd like to thank Jim Propp for introducing us to this problem and for getting us started with the early investigations. Without his REACH program to bring the authors together, this work could not have been done.

\section{References}

[COSW02] Y.B. Choe, J.G. Oxley, A.D. Sokal, and D.G. Wagner. Homogeneous multivariate polynomials with the half-plane property. Preprint, 2002.

[HL72] O.J. Heilmann and E.H. Lieb. Theory of monomer-dimer systems. Comm. Math. Physics, 25:190-232, 1972.

[Kas61] P.W. Kasteleyn. The statistics of dimers on alattice. Physica, 27:1209-1225, 1961.

[KRS96] C. Kenyon, D. Randall, and A. Sinclair. Approximating thenumber of monomer-dimer coverings of a lattice. Journal of Statistical Physics, 83:637-659, 1996.

[Pro01] J. Propp. A reciprocity theorem for domino tilings. The Electronic Journal of Combinatorics, 8:R18, 2001.

[Spe02] D. Speyer. Unpublished note on transfer matrices, 2002. 
[Sta85] R. Stanley. On dimer coverings of rectangles offi xed width. Disc. Appl. Math., 12:81-87, 1985.

[Sta97] R. Stanley. Enumerative Combinatorics, Volume I. Cambridge University Press, 1997.

[TF61] H.N.V. Temperley and M.E. Fisher. Dimer problem instatistical mechanics - an exact result. PhilosophicalMagazine, 6:1061-1063, 1961. 
